\title{
EXIT Analysis of a Soft-Input/Soft-Output Iterative Multiuser Detector
}

\author{
Brad W. Zarikoff and James K. Cavers \\ School of Engineering Science, Faculty of Applied Sciences, Simon Fraser University \\ Burnaby, BC, Canada, V5A 1S6 \\ \{bwzariko@sfu.ca and cavers@sfu.ca\}
}

\begin{abstract}
This paper applies extrinsic information (EXIT) analysis to an internally iterative multiuser detector (IMUD) in serial concatenation with convolutional codes. The focus is overloaded conditions, in which the number of transmitters exceeds the number of receiver antennas. The study seeks to verify if EXIT charts can be used as a design tool under these circumstances. The use of non-Gaussian LLR distributions in performance estimation is discussed. The mutual information of the extrinsic information from IMUD is characterized under a variety of situations. It is shown that the EXIT charts do provide a means to determine both the minimum SNR and necessary number of iterations for convergence. However, estimating the bit error rate from the EXIT chart does not seem to be possible except in special circumstances.
\end{abstract}

Index Terms - MIMO systems, multiuser detection, EXIT analysis, iterative detection.

\section{INTRODUCTION}

$\mathrm{E}$ xtrinsic information transfer (EXIT) charts were introduced in [1] as a useful tool for analyzing convergence in iterative detection of turbo codes. More recently [2], EXIT analysis was applied successfully to serially concatenated multiuser detection (MUD) of coded CDMA signals. The present paper investigates EXIT analysis in a different scenario: serially concatenated multiuser detection of convolutionally coded (CC) narrowband signals, using a new internally iterative MUD component detector that is designed primarily for overloaded conditions (more transmit antennas than receive antennas). We pose three questions: 1) do EXIT charts show whether iteration of this MUD component with the CC converges; 2) do EXIT charts show how many iterations are required; and 3) can the bit error rate (BER) be estimated from the EXIT chart.

Much of MUD research has a general goal of a lowcomplexity, high-performance detector that is capable of operating in overloaded, as well as underloaded (more receive antennas than transmit antennas) conditions. Three approaches to this goal include decision feedback (DF) [3], group detection (GD) [4, 5], and iterative techniques [6-8]. Soft-output MUDs, which include the iterative techniques and

This work was supported by the National Sciences and Engineering Research Council (NSERC). variations on the DF and GD techniques, can be applied in serial concatenation with convolutional codes to provide enhanced BER performance at the cost of complexity.

In [9], an iterative group detector (IMUD) capable of overloaded operation was described. IMUD uses input and output soft decisions, soft cancellation between groups and internal iterations, and involves the randomization of the users within each group between internal iterations. An unusual feature is that group compositions are randomized between internal iterations. This serves to break up correlations introduced by optimal a posteriori probability (APP) extraction within each group.

The EXIT charts developed in [1] will be used to demonstrate the soft decision convergence between IMUD and a convolutional code in an overloaded situation. Where [2] covered the serial concatenated SIC-MUD in a CDMA system detailed in [6], this paper focuses on an internally iterative groupwise detector within a narrowband system. The effects of group size, number of iterations and SNR will be observed.

The rest of the paper is presented as follows. Section II reviews the system parameters. Section III briefly reviews IMUD, focusing on a serial concatenation with a convolutional code. Section IV demonstrates the use of EXIT charts with IMUD. Section V shows the resulting EXIT charts from Monte Carlo simulations. Section VI presents the conclusions.

\section{SYSTEM DESCRIPTION}

The study is done in part with simulations where the channel gain between each transmit and receive antenna is modeled as an independent, Rayleigh flat fading variable (Jakes model). The use of this simple system model allows us to examine the essential multiuser detection problem $[3,7]$.

The system consists of $N$ synchronous transmitters with identical pulse shapes. It is possible for each transmitter to employ multiple antennas, but for simplicity this investigation uses single element transmitters. The receiver uses $M$ antennas, with identical mean SNR at each element. All transmit and receive antennas are uncorrelated. The received samples are matched filtered and sampled once per symbol 
time $T$. Putting aside the time reference, the measurement samples are

$$
\mathbf{y}=\mathbf{H b}+\mathbf{n}
$$

where $\mathbf{y}$ is the length- $M$ received sample vector, $\mathbf{H}$ is the $M \times N$ channel state matrix, $\mathbf{b}$ is the length- $N$ transmitted symbol sequence, and $\mathbf{n}$ is the length- $N$ receiver noise vector. The elements of $\mathbf{H}$ are complex Gaussians with zero mean and variance of $1 / 2$ in each dimension. The fading rate of the channel gains is described with $f_{d} T$, where $f_{d}$ is the Doppler frequency and $T$ is the symbol time. The elements of $\mathbf{n}$ are complex Gaussian with zero mean and a variance of $1 /(2 \gamma)$ in each dimension, where $\gamma$ is the mean SNR per symbol at each antenna.

\section{IMUD IN A CONCATENATED SYSTEM}

IMUD can be broken into 4 parts: group formation, APP extraction, soft cancellation, and iteration. The basis of each will be briefly covered here; see [10] for a more in depth discussion. The concatenation of IMUD with a convolutional code follows.

\section{A. Group Formation}

The groups have the same size, although this is not a necessary condition. Each of the $N_{G}$ groups consists of $G$ users. The received and sampled measurement vector $\mathbf{y}^{(j)}$ as seen by group $j$ after soft cancellation from the previous groups can be rewritten

$$
\begin{aligned}
\mathbf{y}^{(j)} & =\mathbf{H}_{j} \mathbf{b}_{j}+\sum_{i=1}^{j-1} \mathbf{r}_{i}+\sum_{i=j+1}^{N_{G}} \mathbf{H}_{i} \mathbf{b}_{i}+\mathbf{n} \\
& =\mathbf{H}_{j} \mathbf{b}_{j}+\mathbf{u}^{(j)}
\end{aligned}
$$

where $\mathbf{H}_{j}$ and $\mathbf{b}_{j}$ are the channel gains and data symbols in group $j$ and $\mathbf{r}_{i}$ is the residual error component after cancellation of group $i$. $\mathbf{u}^{(j)}$ is the undesired portion of $\mathbf{y}^{(j)}$. The membership of group $j$ is determined on the basis of error variance in the MMSE estimation of the remaining $N-(j-1) G$ users, according to

$$
\Sigma_{e}^{2}=\operatorname{diag}\left(\mathbf{I}-\mathbf{H}^{(j) \dagger}\left(\mathbf{H}^{(j)} \mathbf{H}^{(j) \dagger}+\frac{1}{2 \gamma} \mathbf{I}\right)^{-1} \mathbf{H}^{(j)}\right)
$$

where $\mathbf{H}^{(j)}$ refers to the matrix $\mathbf{H}$ with all users in groups 1 to $j-1$ removed. The $G$ users with the lowest value are then selected to from the $j^{\text {th }}$ group. It must be noted that this error variance minization (EVM) criterion is only used for the $N_{G}$ groups of the first iteration of IMUD. Groups are formed randomly in subsequent iterations, as explained in Section IIID.

\section{B. Group APP Extraction}

Symbol detection in IMUD is accomplished using the group APP extractor (GAPPE). It is optimal for an individual group, where the joint APP's are found using the conditional probability of $\mathbf{y}^{(j)}$ and any a priori information available. Assuming that the undesired variable $\mathbf{u}^{(j)}$ in (2) is Gaussian distributed allows us to find the log of the conditional probability density to be

$$
\begin{array}{r}
\log \left(\operatorname{Pr}\left(\mathbf{y}^{(j)} \mid \mathbf{b}_{j}\right)\right)=-\log \left(\pi^{M}\left|\mathbf{R}_{\mathbf{u}}^{(j)}\right|\right)+ \\
\left(\mathbf{y}^{(j)}-\mathbf{H}_{j} \mathbf{b}_{j}\right)^{\dagger} \mathbf{R}_{\mathbf{u}}^{(j)-1}\left(\mathbf{y}^{(j)}-\mathbf{H}_{j} \mathbf{b}_{j}\right)
\end{array}
$$

where

$$
\mathbf{R}_{\mathbf{u}}^{(j)}=\sum_{i<j} \mathbf{R}_{\mathbf{r} i}+\sum_{i>j} \mathbf{H}_{i} \mathbf{H}_{i}^{\dagger}+\frac{1}{2 \gamma} \mathbf{I}_{M}
$$

$\mathbf{R}_{\mathbf{r} i}$ is the covariance matrix for the detected users, and is defined in Section III- $C$. The first term in (4) can be neglected since it is the same for all users within the $j^{\text {th }}$ group. The conditional probability from (4) is then marginalized over all possible transmitted symbols using a prioris to find the $a$ posteriori probability (APP). It is worth noting here that the sphere decoding algorithm can be used to reduce the complexity of the GAPPE [10]. The a prioris are denoted $\operatorname{Pr}^{(a)}\left(b_{m j}\right)$ for the $m^{\text {th }}$ user of the $j^{\text {th }}$ group. The $n^{\text {th }}$ users loglikelihood ratio, denoted $\mathbf{L}_{I M U D}\left(b_{n j}\right)$, is

$$
\begin{gathered}
\mathbf{L}_{\text {IMUD }}\left(b_{n j}\right)=\log \left(\operatorname{Pr}\left(\mathbf{y}^{(j)}, b_{n j}=+1\right)\right)- \\
\log \left(\operatorname{Pr}\left(\mathbf{y}^{(j)}, b_{n j}=-1\right)\right)
\end{gathered}
$$

The joint probability is created in (6) using (4) and the $a$ prioris. However, the max-log-MAP algorithm in [11] can be used to approximate the $\log$ of a sum of exponentials and reduce the complexity. If desired, the sign of $\mathbf{L}_{I M U D}\left(b_{n j}\right)$ can be used for a hard decision.

\section{Soft Cancellation}

Between each group detection and iteration of IMUD, the first and second order statistics of each user are calculated and used for interference cancellation. For BPSK, the a posteriori mean and variance of the $n^{\text {th }}$ bit of the $j^{\text {th }}$ group is

$$
\begin{aligned}
& \mu_{n j}^{(o)}=\operatorname{Pr}^{(o)}\left(b_{n j}=1\right)-\operatorname{Pr}^{(o)}\left(b_{n j}=-1\right) \\
& \sigma_{n j}^{(o) 2}=1-\mu_{n j}^{(o) 2}
\end{aligned}
$$

Representing a full group's a posteriori mean as $\boldsymbol{\mu}_{j}^{(o)}$, the interference cancellation is accomplished by subtracting $\mathbf{H}_{j} \boldsymbol{\mu}_{j}^{(o)}$ from the measurement vector to create $\mathbf{y}^{(j+1)}$. The covariance matrix $\mathbf{R}_{\mathbf{r} i}$ in (5) is based on the assumption that the errors are uncorrelated, which allows the matrix to simply have the diagonals updated with the error covariance from (8). This covariance matrix is only updated upon the completion of each group's GAPPE. The APPs are forwarded to the next iteration, and are used both as a priori probabilities in the joint probability of (6) and for interference cancellation. 


\section{Iteration}

To ensure that each iteration makes full use of a prioris and the group structure, all groups are randomized after the first iteration. The EVM based ordering criterion for the first iteration ensures that IMUD starts with a low BER, but it is not necessary to the final error performance; it will simply take more iterations to achieve the same BER [10]. The randomization provides the GAPPE with a different set of user correlations than those that were used to create the a priori information. It is analogous to interleaving in the case of turbo codes.

In contrast to most iterative schemes, the removal of $a$ prioris from the full APPs is not performed between IMUD iterations [9]. Simulations show that in the absence of interference cancellation, the removal of a priori information causes error floors. This is thought to be a product of correlation between the a prioris and the extrinsic information provided by GAPPE.

\section{E. Serial Concatenation}

To accommodate coded multiuser bit streams, we can concatenate IMUD with a set of single-user convolutional code (CC) soft-input/soft-output (SISO) decoders and exploit the soft decisions. In this combination, IMUD is a SISO multiuser detector with no temporal memory, and the $\mathrm{CC}$ decoders are single-user SISO detectors that exploit the memory in their code trellises. This is similar to that proposed in [7], with the channel assumed to be perfectly known. It is also possible to incorporate trellis-based space-time coding in this framework, although we have not done so in our simulations.

Figure 1 shows the concatenated system. CC encoders transform the finite-length data streams $u_{n}(t)$ to coded streams $c_{n}(t)$, then independent interleavers produce the signals $s_{n}(t)$ transmitted over the shared channel. The receiver matched filter outputs at time $t$ are components of the length- $M$ vector $\mathbf{y}(t)$, and those vectors enter the serially concatenated iterative decoder. That decoder alternates between: 1) applying IMUD, possibly with non-equal a priori information from the $\mathrm{CC}$ decoders, in order to extract the APPs for all times $t$; and 2) deinterleaving the APPs and running the $\mathrm{CC}$ decoders on them independently by user to produce improved a posteriori information for re-interleaving and delivery to IMUD.

\section{F. Information Flow}

The flow of the concatenated system is shown graphically in Figure 2. For the first iteration, IMUD begins by creating preliminary probabilities for the transmitted symbols as $\mathbf{L}_{I M U D}^{(1)}\left(s_{n}\right)$; when IMUD is used simply as a multiuser detector, these probabilities are then put through a hard decision. The IMUD LLR consists of a priori and extrinsic

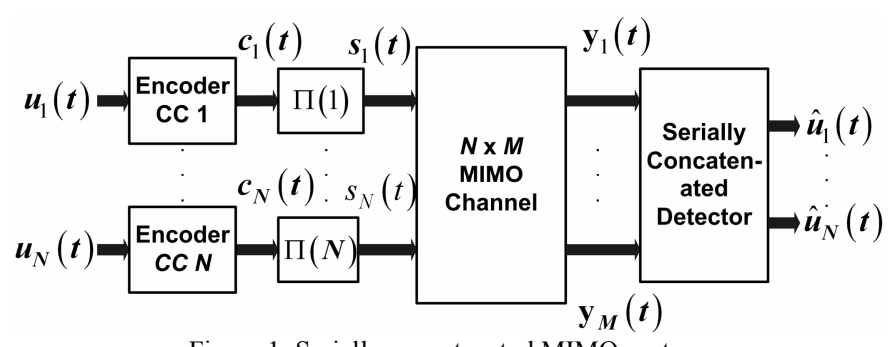

Figure 1. Serially concatenated MIMO system.

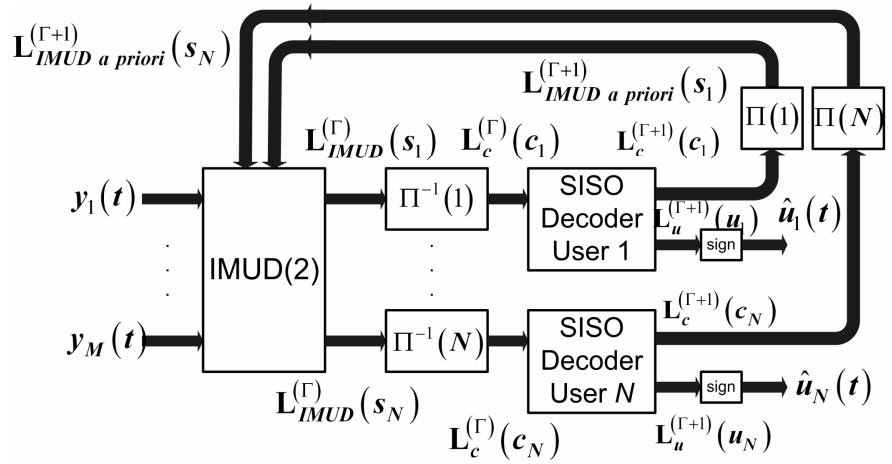

Figure 2. Serially concatenated receiver structure.

information, and originates from the group APP extraction in each of the iterations within IMUD. For the $\Gamma^{\text {th }}$ iteration of the concatenated system, the a priori probabilities $\mathbf{L}_{I M U D \text { a priori }}^{(\Gamma)}\left(s_{n}\right)$ are removed from $\mathbf{L}_{I M U D}^{(\Gamma)}\left(s_{n}\right)$ before the LLRs are deinterleaved and fed into the CC decoder.

The CC decoder processes the deinterleaved LLRs $\mathbf{L}_{c}^{(\Gamma)}\left(c_{n}\right)$ using the method described in [12]. Since the CC decoder is strictly soft-input, the CC output LLRs $\mathbf{L}_{c}^{(\Gamma+1)}\left(c_{n}\right)$ and $\mathbf{L}_{u}^{(\Gamma+1)}\left(u_{n}\right)$ consist solely of the a priori LLRs from IMUD and any extrinsic information made available through the decoding process; there is no channel information in the LLRs at this point. The a priori information of the coded bits is easily removed at the output. Finally, the extrinsic information associated with the coded bits at the output of the CC block is interleaved, and is forwarded to IMUD for the next iteration.

For the second iteration, the only difference is that the $a$ priori information for IMUD is now supplied by the coded bit LLRs from the CC block. When a decision is desired, a hard decision is applied to the LLRs for the uncoded bit $\mathbf{L}_{u}^{(\Gamma+1)}\left(u_{n}\right)$, which is available at the output of the CC block.

\section{EXIT ANALYSIS}

The LLRs at the output of each block can be used to characterize the speed of convergence and performance of a given iterative system. It is shown in [1] that EXIT charts can predict the progress of iteration of individual SISO blocks in Turbo decoding using the mutual information between the data sequence and the LLRs. Each block is characterized individually in terms of extrinsic information curves; the convergence properties are revealed when a block is compared 
to its companion with which it will be trading LLRs. [1] demonstrates that mapping the transfer characteristics from a single-user TURBO detector on a single chart allows the observer to determine whether convergence is possible and how many iterations are necessary for a given performance level.

\section{A. Transfer Curves}

The mutual information between the LLRs output by the block and the known transmitted symbols are used to form the transfer curves. The expression for the mutual information is dependent on the pdf of the LLRs. As noted in [1] for a BPSK signal, the mutual information is defined as

$$
\begin{gathered}
\mathrm{I}\left(b_{n j}\right)=\mathrm{I}\left(\mathbf{L}^{(x)}\left(b_{n j}\right) ; b_{n j}\right)=\frac{1}{2} \sum_{b_{n j} \in\{ \pm 1\}} \int_{-\infty}^{+\infty} f\left(\mathbf{L}^{(x)} \mid b_{n j}\right) \\
\cdot \log _{2}\left(\frac{2 \cdot f\left(\mathbf{L}^{(x)} \mid b_{n j}\right)}{f\left(\mathbf{L}^{(x)} \mid b_{n j}=+1\right)+f\left(\mathbf{L}^{(x)} \mid b_{n j}=-1\right)}\right) d \mathbf{L}
\end{gathered}
$$

where $x$ may refer to either a priori or a posteriori probabilities, and $\mathrm{I}\left(b_{n j}\right) \in[0,1]$. If we have the distribution of the LLRs and the vector of transmitted symbols, then we can determine the mutual information and thus the transfer curve. If the LLRs are Gaussian distributed, the expression for mutual information can be greatly simplified.

In the case where the LLRs are not Gaussian distributed, [2] makes the observation that a histogram of $\mathbf{L}^{(x)}\left(b_{n j}\right)$ can be used to estimate $f\left(\mathbf{L}^{(x)} \mid b_{n j}\right)$ and calculate the mutual information. [2] also reports on the effect of block size on the transfer characteristics, which is especially important in block oriented systems such as our serially concatenated system.

For our simulations, the extrinsic mutual information in the EXIT curves was calculated using consistent Gaussian distributed a priori LLRs.

\section{B. Error Rate Estimation}

In the case of a single-user BPSK constellation and a Gaussian LLR distribution, an estimate of the error rate can be calculated using the statistics of the distribution and the complementary error function [1]. This is extended to a single user in a Rayleigh fading channel in the same paper. In [2], the LLR distribution for two DF MUDs in a multipath fading environment are assumed to be Gaussian for the case of BER estimation with very good results. As shown below, this is not the case with all detectors.

To start our analysis of the LLR distribution of IMUD, we examined the LLRs of the optimum soft-output MUD [13] in a static multipath channel with the application of the max-logMAP (detailed in [11]). This can be considered a special case of IMUD where the number of groups $N_{G}=1$. Max-logMAP was utilized in order to simplify the analysis of the LLR distribution since the marginalization operation in the optimum technique made analysis difficult at best. The reason for this examination was to characterize the LLR distribution. If a one-to-one relationship can be established between the statistical parameters of the LLRs and the mutual information between the LLRs and the data, then the error rate can be calculated using the EXIT chart. In [1], this was accomplished by noting that the LLRs in detection of turbo codes are approximately Gaussian, and invoking a consistency condition that made the mean equal to half the variance. In the optimum MUD case, we have calculated separately that the single user's LLR after detection with the max-log-MAP approximation has a conditional mean

$$
E\left[\mathbf{L}^{(e x t)}\left(b_{n j}\right) \mid b_{n j}, \mathbf{H}_{n j}\right]=8 \cdot \gamma \cdot b_{n j}\left(\sum_{k=1}^{M}\left|h_{k, n j}{ }^{2}\right|\right)
$$

This is a good, intuitive value as it directly shows the diversity benefit of added receive antennas and the effect of SNR. The experimental results show that the single-user LLRs do follow an approximately Gaussian distribution, with the mean agreeing with (10). However, the experimental LLR variance did not equal twice the mean, as the consistency condition would require, and we did not observe an alternative one-toone link between mean and variance. In fact, the actual IMUD LLRs exhibited modalities. This shows that without further work the BER is not obtainable solely with the mutual information in the case of joint detection, and therefore IMUD.

\section{Effects of Finite Block Length}

Since digital communications links use blocks of data for transmission, block estimates (not asymptotic results) will be used to gauge the effectiveness of a given technique. However, a finite block suffers from statistical variations; in our case of mutual information, the effect is a variance in the extrinsic information curve. [2] documents the effects of finite block length and how to use the second order statistics to determine the minimum SNR for convergence. The variance of the EXIT curves is not presented on the graphs. However, since the curves are generated using 500 symbol blocks, the curves consist of an average over many simulations and contain some variation, especially between the EXIT curves and the trajectories.

\section{RESULTS}

IMUD transfer curves in a variety of configurations were found with the aid of the techniques reviewed in Section III. Blocks of 500 symbols were transmitted over the multipath fading channel detailed in Section II. The a priori LLRs used during generation of the EXIT curves were Gaussian distributed LLRs, with the statistics based on the input information level. The extrinsic LLRs $\mathbf{L}^{(e x t)}\left(b_{n j}\right)$ were used to estimate $f\left(\mathbf{L}^{(x)} \mid b_{n j}\right)$ in (9). All user LLRs were averaged 
in the histogram since they all have identical mean SNR at the receive antennas. The rate $2 / 3$ convolutional code (CC) in the simulations is non-systematic with generators $(2,7),(7,5)$ and $(7,2)$ and constraint length 3 ; its curve was generated by uncorrelated samples, equivalent to perfect interleaving. For IMUD, the group configurations are specified by the triple $\left(M, N_{G}, G\right)$; for example, $(4,6,2)$ indicates 4 antennas and 6 groups of 2 users each (for a total of 12 users). Also, the notation IMUD(iteration) denotes the number of internal iterations.

The effect of the number of iterations on IMUD is demonstrated in Figures 3 and 4. The overloaded system consists of 4 receive antennas and 6 users, in a $(4,3,2)$ configuration. Figure 3 shows the BER versus SNR curves of the system for $\Gamma=1,2$ and 3 iterations between $\operatorname{IMUD}(2)$ and the CC with $f_{d} T=0.01$. The single-user curve is given as a reference. At a BER of $10^{-3}$, the second iteration produces a $2 \mathrm{~dB}$ improvement from the first iteration. Further iterations have diminishing returns. The performance at a BER of $10^{-3}$ after the third iteration is only $1 / 4 \mathrm{~dB}$ poorer than that of the performance of a coded single-user. For comparison, we note that the net information rate of six users, each with the rate $2 / 3$ code, is the same as that of four uncoded users. With four antennas, JML of four uncoded users requires an SNR of 4.5 $\mathrm{dB}$ to achieve a BER of $10^{-3}$ [9], compared with approximately $-1.5 \mathrm{~dB}$ for the concatenated detection of the six coded users at the same net throughput.

In Figure 4, the transfer characteristic for IMUD and the inverse transfer characteristic for the $\mathrm{CC}$ are plotted along with the mutual information trajectories of the iteration. Note that the inverse of the CC EXIT curve was plotted to demonstrate the flow of extrinsic information from block to block (since the extrinsic information from one block is the $a$ priori information into the next block). For a fading rate of $f_{d} T=0.01$ and an SNR of $-2 \mathrm{~dB}$, convergence is achieved within 3 iterations; this is verified in Figure 3. The deviation from the EXIT curve can be explained by the increasing correlation of the a prioris and APPs as the iterations proceed. The trajectories are made up of an average of 450 blocks in order to deal with the EXIT curve variance [2]. For static fading, trajectories (Traj.) are shown for $\operatorname{IMUD(2).~Since~}$ IMUD is a symbol-by-symbol detector, the limitation for this channel is the CC. The interleaver is unable to provide time diversity in this case, and thus performance of the $\mathrm{CC}$ deteriorates. The static case trajectory $\left(f_{d} T=0\right)$ shows that the CC curve shifts upwards.

Figure 4 also shows the effect of iterations within IMUD. The upward shift of the EXIT curve between $\operatorname{IMUD}(2)$ and IMUD(1) reflects the increase in diversity demonstrated in [9]. Finally, Figure 4 demonstrates the effect of an unsuccessful convergence due to low SNR; at $-4 \mathrm{~dB}$, IMUD(2) crosses the

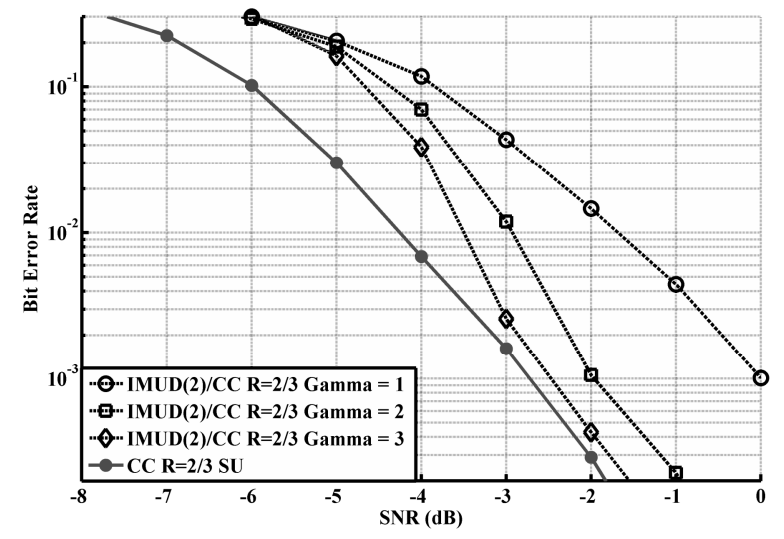

Figure 3. BER curves of overloaded $(4,3,2)$ concatenated system with $\operatorname{IMUD}(2)$ for $\Gamma=1,2$, and 3 with a fading rate $f_{d} T=0.01$.

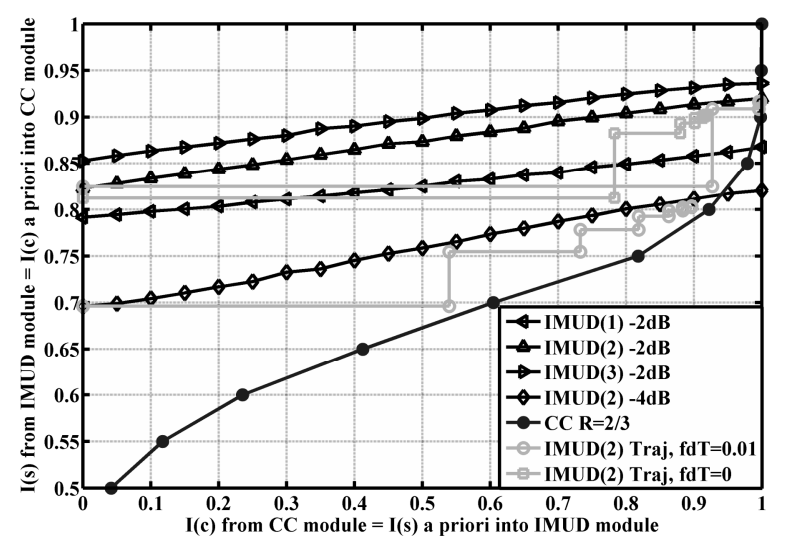

Figure 4. EXIT curves and trajectories of overloaded $(4,3,2)$ concatenated system at an SNR of $-2 \mathrm{~dB}$ and $-4 \mathrm{~dB}$.

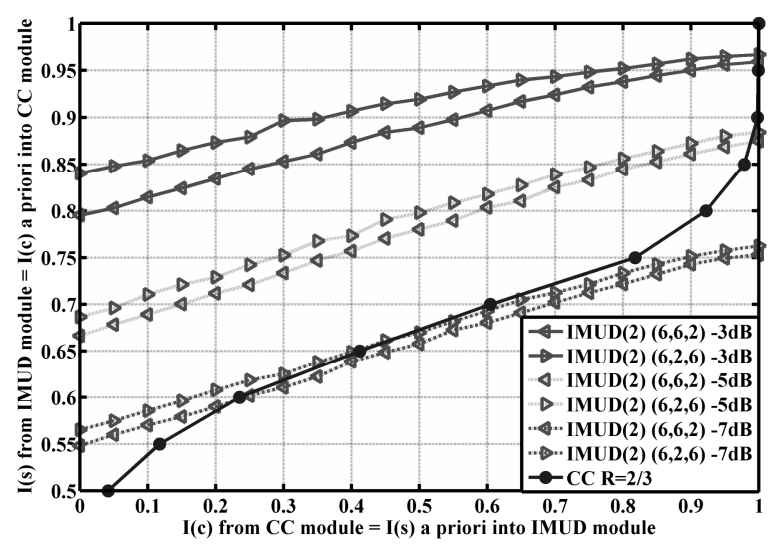

Figure 5. Effect of SNR on overloaded 6 receive antenna, 12 user system.

CC EXIT curve at a mutual information level of slightly more than 0.9. The trajectory shows that even after $\Gamma=7$ iterations, the concatenated system achieves an extrinsic information level of less than 0.9. The failure to converge completely to the intersection of the curves is hypothesized to be due to the finite block size effects.

The Gaussian assumption cannot be made for BER estimation for this concatenated case. The reason is due to the 
LLR distribution of IMUD. The symbol decisions are made on the full APP at the output of the CC decoder, which consists of the almost Gaussian distributed extrinsic APPs from the decoder and possibly non-Gaussian a prioris from IMUD. In the static case, the IMUD extrinsic APPs are almost Gaussian, as noted in Section IV- $B$. However, estimation is not possible using the Gaussian methods in $[1,2]$ since there is no one-to-one relationship between the extrinsic information and the mean and variance parameters of the LLR distribution.

Figure 5 shows transfer curves for a 6 receive element, 12 user system with SNR values of $-3 \mathrm{~dB},-5 \mathrm{~dB}$, and $-7 \mathrm{~dB}$. As would be expected, a drop in SNR results in a drop in the EXIT curve. The gain between IMUD in a $(6,2,6)$ and $(6,6,2)$ configuration also drops with the SNR. This is thought to be resulting from the increased signal corruption from very low SNR.

The EXIT approach to convergence analysis also suggests hybrid approach schemes that may reduce computational complexity and/or improve converged BER. For example, recall that the computational load of $\operatorname{IMUD}(2)$ is almost twice that of IMUD(1), and that IMUD(3) has almost three times the load of IMUD(1). From Figure 4, we see that the first iteration $(\Gamma=1)$ could be accomplished with $\operatorname{IMUD}(1)$, the second $(\Gamma=2)$ with $\operatorname{IMUD}(2)$ and so on, thereby reducing computation in earlier iteration. As another example, both the computational load and the amount of output extrinsic information in an $\left(M, N_{G}, G\right)$ IMUD increase as the number of groups $N_{G}$ decreases and the number of users per group $G$ increases, for a fixed number of users $N_{G} G$. Again, earlier iterations of the concatenated system could use smaller group sizes. Further testing is necessary to confirm these optimizations.

\section{CONCLUSIONS}

The extrinsic information of iterative techniques is valuable as a means to analyze convergence of multiuser detection in antenna overload conditions without relying completely on full simulation and their BER curves. We have determined that 1) the EXIT charts can be used to determine the level of mutual information after convergence of a concatenated system consisting of iterative MUDs and 2) that the number of iterations necessary for the convergence can also be determined from the charts. However, it was found that the mutual information value is not sufficient to estimate the BER because the IMUD LLRs were shown not to be consistent Gaussian.

The EXIT curves for IMUD were calculated for two overloaded cases. A concatenated system consisting of IMUD and a rate $2 / 3$ SISO convolutional code was used to show the effects of the iterations. Even in heavy overload conditions -
12 users received with only 6 receive antennas - EXIT analysis was sufficient to determine the number of iterations to convergence and the mutual information between outputs and the data sequence obtained at convergence.

EXIT curves also showed their value by suggesting new hybrid strategies, in which components with different qualities and computation loads could be used on different iterations.

\section{REFERENCES}

[1] S. ten Brink, "Convergence behavior of iteratively decoded parallel concatenated codes," IEEE Trans. Commun., vol. 49, pp. 1727-1737, 2001.

[2] K. Li and X. Wang, "EXIT chart analysis of turbo multiuser detection," IEEE Trans. Wireless Commun., vol. 4, pp. 300-311, 2005.

[3] P.W. Wolniansky, G.J. Foschini, G.D. Golden and R.A. Valenzuela, "V-BLAST: an architecture for realizing very high data rates over the rich-scattering wireless channel," in IEEE URSI Int. Symp. on Signals, Systems and Electronics, 1998, pp. 295-300.

[4] E.A. Fain and M.K. Varanasi, "Diversity order gain for narrow-band multiuser communications with pre-combining group detection," IEEE Trans. Commun., vol. 48, pp. 533-536, 2000.

[5] B.K. Ng and E.S. Sousa, "On bandwidth-efficient multiuser-space-time signal design and detection," IEEE Journal on Select. Areas in Commun., vol. 20, pp. 320-329, 2002.

[6] X. Wang and H.V. Poor, "Iterative (turbo) soft interference cancellation and decoding for coded CDMA," IEEE Trans. Commun., vol. 47, pp. 1046-1061, 1999.

[7] M. Sellathurai and S. Haykin, "TURBO-BLAST for high-speed wireless communications," in IEEE Wireless Commun. and Networking Conf. 2000, pp. 315-320.

[8] J. Luo, K.R. Pattipati, P.K. Willett and F. Hasegawa, "Near-optimal multiuser detection in synchronous CDMA using probabilistic data association," IEEE Commun. Lett., vol. 5, pp. 361-363, 2001.

[9] B.W. Zarikoff, "Investigation of an Iterative Groupwise Soft Input/Output Multiuser Detection Algorithm," Theses (School of Engineering Science) / Simon Fraser University, 2004.

[10] B.W. Zarikoff, J.K. Cavers and S. Bavarian, "An Iterative Groupwise Multiuser Detector for Overloaded MIMO Applications," unpublished.

[11] P. Robertson, E. Villebrun and P. Hoeher, "A comparison of optimal and sub-optimal MAP decoding algorithms operating in the log domain," in Proc. IEEE ICC, 1995, pp. 1009-1013 Vol. 2.

[12] S. Benedetto, D. Divsalar, G. Montorsi and F. Pollara, "A soft-input soft-output APP module for iterative decoding of concatenated codes," IEEE Commun. Lett., vol. 1, pp. 22-24, 1997.

[13] B.M. Hochwald and S. ten Brink, "Achieving near-capacity on a multiple-antenna channel," IEEE Trans. Commun., vol. 51, pp. 389$399,2003$. 